\title{
A Synchronous and Closed-Loop Architecture of BCI-Based Rehabilitation System for Stroke with Robot and Virtual Reality
}

\author{
Chuanqi Tan, Fuchun Sun*, Wenchang Zhang and Shaobo Liu \\ Department of Computer Science and Technology, Tsinghua University, Beijing, China \\ ${ }^{*}$ Corresponding author
}

\begin{abstract}
In this paper, we proposed a novel architecture of a scalp electroencephalography (EEG) based rehabilitatoin system via brain computer interface with robotic and virtual reality devices. More and more attentions had been taken to the brain computer interface, because it is the only way for some special patients to communicate with the outside world.EEG has show significant potential as a non-invasive way for BCI. But it is very hard to decode useful information from EEG signals in a reliable and efficient way, and also very difficult to get desiable rehabilitation result by current rehabilitation system. To this end, we propose an integrated EEG-driven rehabilitation system with assistive robotic and virtual reality feedback for some stroke patients. The framework of our method contains follow steps: signals accquire, signals preprocess; feature extract; classificatoin and feedback to human. Many advance algorithm has been surveryed and combined to accquire a better result. We use common spatial pattern as the spatial feature of signals, and convert signals to spectral via fast fourier transform. With the spatial and spectral features, we use support vector machine to classify these features and reduce final classification result by a fusion algorithm. At last, the classification result has been used to control robot and virtual reality devices. This synchronous and closed-loop rehabilitation system can achieve benefits for some peoples who suffer from stroke.
\end{abstract}

Keywords-component; EEG; BCI; close-loop; robot; virtual reality; rehabilitation system

\section{INTRODUCTION}

Human brain plays akey role in controlling our body. It can be said that the human brain is the most complex organ in the world. For some special patients who suffering from disorders such as stroke, it is very important to provide a communication method to transfer brain messages except from the normal output pathway [1]. EEG signals are selected as the input by most BCI systems because of the natural and non-invasive approach when the signals are recorded, also because the EEG equipment are becoming more available on the public market.EEG is recorded by electrodes which reflects regular electric action of brain cells groups, which is a very important electrical activity of the pyramidal cortical cells. Motor imagery, which is a mental process, can be treated as an individual rehearses or simulates a given motor act. By thinking about a motor act, people can produce corresponding motor-related EEG patterns. When EEG signals had been decoded, the patterns can be translated to commands to control external equipment [2].For stroke patients, it is useful to use
BCI-based system to control rehabilitation equipment to assist them in performing rehabilitation exercises.

BCI-based systems can be divided into four categories by the pattern of brain activity, such as slow cortical potentials (SCPs), P300 component of event related potentials (ERPs), steady-state visual evoked potentials (SSVEPs) and eventrelated desynchronization/synchronization (ERD/ERS) [3]. The brain activity of ERD/ERS has been widely used as the sources during motor imagery in BCI.BCI-based system can be advised as a complex pattern recognition system [4], which the framework of BCI contains the parts of signal acquisition, preprocess, feature extract, classification algorithm, control signal to external devices and feedback. The goal of feature extraction step is describing the EEG signals distinctly. The classification step is focus at assigns a class label to the feature vector extracted from the EEG signal.

In the past decades, researches around the world tried to decode EEG signals correctly. A number of methods have been proposed to design an efficient feature extraction algorithm in EEG-based BCI. Such as band power (BP), power spectral density (PSD) [5], autoregressive (AR) [6], iterative twodimensional nearest feature line(INFL). In recent years, the Common Space Model (CSP) [7] has proven to be an effective feature of EEG signals and has been proposed for a number of related work such as CSSP, CSSSP, FBCSP, WCSP, Stationary Common Spatial Patterns and SCSSP. The works of these pioneers provides a very big inspiration and help for our work.

In past years, they are many classification algorithms used to design BCI systems. They are divided into five different categories [8]: linear classifiers such as Discriminant Analysis (LDA) and Support Vector Machine (SVM), neural networks such as MultiLayer Perceptron (MLP), nonlinear bayesian classifiers such as Bayes quadratic and Hidden Markov Model (HMM), nearest neighbor classifiers such as $k$ Nearest Neighbors (kNN)and combinations of classifiers.

A recent trend, however, is to use several classifiers, aggregated in different ways. The classifier combination strategies offen used in BCI applications are boosting, voting, stacking. The main advantage of such techniques is that a combination of similar classifiers is very likely to outperform one of the classifiers on its own. Actually, combining classifiers is known to reduce the varianceand thus the classification error. 
There is a lot of challenges in EEG-based BCI. In this paper, us contributes is a more effective feature extraction and classification algorithm, furthermore, contain a fusion algorithm to fuse multiply classifier for more accuracy in final classification results. In addition, designed the architechture of BCI-based rehabilitation system use robot and virtual reality for stroke patients. The rest of this paper is organized as follows. The EEG classification and fusion algorithm proposed by us will introduce in Section 2. In Section 3, our BCI-based rehabilitation system has been introducted. At last, conclusions are given in Section 4.

\section{METHODS}

In our approach, the whole approach is roughly divided into four main stages. First, as with other bioelectrical signals, the EEG signal is flooded with a lot of noise, and the first stage of preprocessing is mainly used for filtering the EEG signal. This step is very important. The second stage, called feature extraction and classification, extracts the spatial and frequency characteristics of the EEG signal and uses SVM to classify it. The third stage is the fusion phase, which combines the results from the previous phase. The final phase includes an iterative strategy to optimize the parameters from the training data.

\section{A. preprocess}

The signals of the different channels on the EEG signal collector show the electrical signal strength of the different functional areas of the brain. We choose channels to avoid channels with very low signal-to-noise ratios, so that we can have some positive effects in the next classification phase. We then select the middle portion of the signal segment to obtain a purer EEG signal because it is susceptible to interference from other signals during the imaging start and end periods.

Then we apply fastICA algorithm [9] to blind source separation to remove the noise of other parts of the body, but also to achieve the effect of dimension reduction, the latter aspects of the deal. Independent Component Analysis (ICA) is a blind source separation algorithm used to find basic factors or components from a combination of data. FastICA is a widely used ICA algorithm, which is more efficient than the traditional gradient descent method. In the final preprocessing, we simply use some low-pass filter [10], which eliminates the high-order part of the signal, which is often easier to noise.

\section{B. Feature extract}

To improve the accuracy of classification, we combine the common spatial pattern as the spatial information and statistical feature of fast Fourier transform as the spectral information to get a better expressive characteristic.

For extract spatial information, the raw EEG data of a single trial is represented as an $\mathrm{N}^{*} \mathrm{~T}$ matrix E, where $\mathrm{N}$ is the number of channels and $\mathrm{T}$ is the number of samples per channel. At the beginning of this feature extract step, we minus mean in every channels to convert the signal vibration near zero. The normalized spatial covariance of the EEG can be obtained from:

$$
C=\frac{E E^{\prime}}{\operatorname{trace}\left(E E^{\prime}\right)}
$$

The spatial covariance $\overline{C_{x}}$ to the two distributions to be separated is calculated by averaging over the trials of each group. The composite spatial covariance is given as

$$
C_{c}=\overline{C_{1}}+\overline{C_{r}}=U_{c} \lambda_{c} U_{c}{ }^{\prime}
$$

where the second equal is the factor of $\mathrm{C}_{\mathrm{c}}$. Sort eigenvalues in descending order and do whitening transformation.

$$
P=\sqrt{\lambda_{c}^{-1}} U_{c}^{\prime}
$$

if $\overline{C_{1}}$ and $\overline{C_{r}}$ are transformed to $\mathrm{S}_{1}, \mathrm{~S}_{\mathrm{r}}$ by

$$
S_{k}=P \overline{C_{k}} P^{\prime}
$$

Then $\mathrm{S}_{1}$ and $\mathrm{S}_{\mathrm{r}}$ share common eigenvectors $\mathrm{B}$ with the sum of two corresponding eigenvalues is always one. The eigenvector with largest eigenvalue for $S_{1}$ has the smallest eigenvalue for $S_{r}$ and vice versa. This property makes the eigenvectors B useful for classification of the two distributions. $\mathrm{E}$ is

With the projection matrix $W=B^{\prime} P^{\prime}$, the decomposition of

$$
Z=W E
$$

where $W^{-1}$ are the common spatial patterns and can be seen as time-invariant EEG source distribution vectors.

For extract spectral information, fast Fourier transform (FFT) has been apply to every fragment in every channels [11]. We choose the coefficients in the Fourier transform to represent these EEG signals, and then perform some mathematical operations on these coefficients, such as averaging and variance. These statistical results are then used to characterize these EEG signals in the frequency domain value.

\section{Classification}

The classification step in a BCI system intend to label the feature vector. We use support vector machine (SVM) to classify the spatial information and spectral information [12]. The purpose of SVM is to use hyperplanes to separate the training feature vectors to get the largest distance between support vector and separate hyperplanes.

SVM has been proved to be a very good classifier when the training data is small. In contrast, classifiers such as neural networks require a large amount of training data, and so much training data is not possible in BCI Get it. This is why SVM can play an important role in BCI. 
To overcome limitations and disadvantages of conventional EEG classification algorithms, we fuse many classification results in different aspects based on a Bayesian-decision like algorithm to achieve better results [13]. Based on the classical Bayesian theory. Then use follow equal to determine the final class as the fusion results.

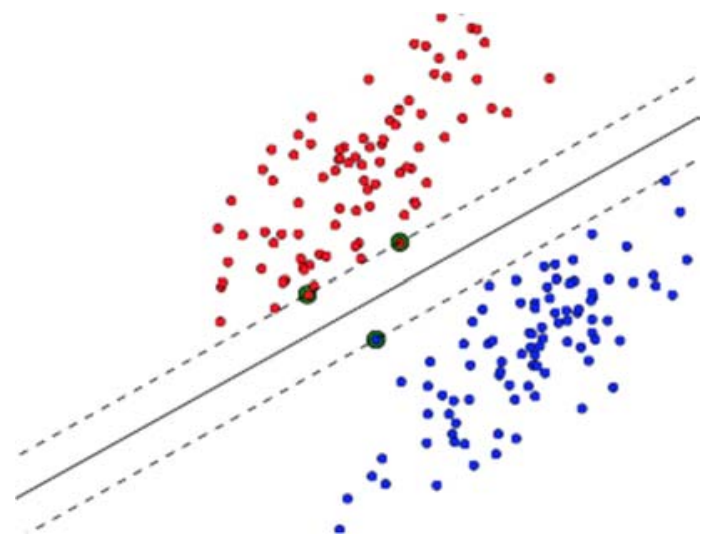

FIGURE I. SUPPORT VECTOR MACHINE

$$
\begin{aligned}
\hat{j} & =\underset{j \in C}{\operatorname{argmax}} P\left(\omega_{j} \mid x_{1}, x_{2}, \ldots, x_{R}\right) \\
& =\underset{j \in C}{\operatorname{argmax}} P\left(\omega_{j}\right) \sum_{i=1}^{R} W_{i} P\left(\omega_{j} \mid x_{i}\right)
\end{aligned}
$$

\section{Parameter optimization}

We design a algorithm try to find out the best parameters in the framework by recursive, which do the follow steps in every iterator. First, split the train-set to a small train-set and a validation-set. Second, choose only one parameter changing sequentially and fix the other parameters. Third, calculate the results based on validation-set while the selected parameter changing and find out the best value of selected parameter in this iterative. The algorithm terminated while the target number was reached. The pseudo-code of algorithm are shows in Algo. 1.

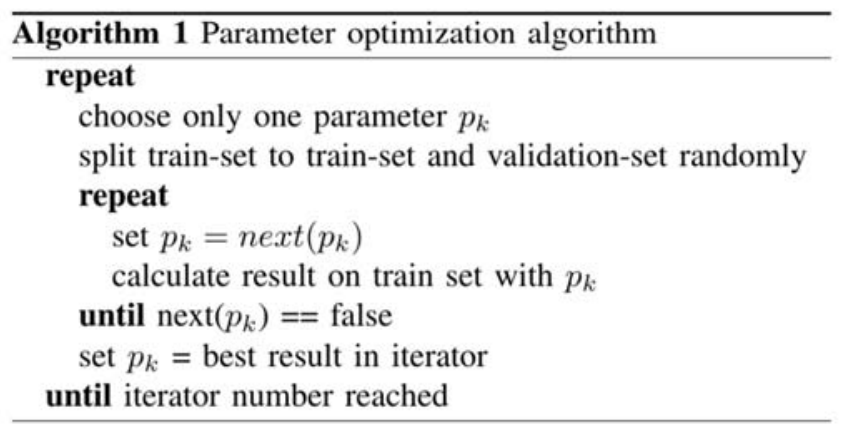

\section{RESULTS}

As before research, an effective rehabilitation system need to have follow charaticstics: the user realizes the movement; the user imagines the movement; the user observes the movement [14]. Follow this idea, we apply our methods discuss aboved to decode the mind of subject when their blind and flex elbow.

We choose a baxter robot and a virtual reality display device as the feedback of our rehabilitation system. Baxter robotic is a double arm robot made by rethink robotics, these arms can be control in programmatic. After we get control command in before steps, the baxter robot follow the command by move arms. Furthermore, a virtual reality device will display the feedback information to the human eyes. By receive the feedback information, the subject will change their mind immediately. The rehabilitation system can adjust paramters automation via these information cycles. By finished this, our rehabilitation system is a close-loop rehalibitation system.

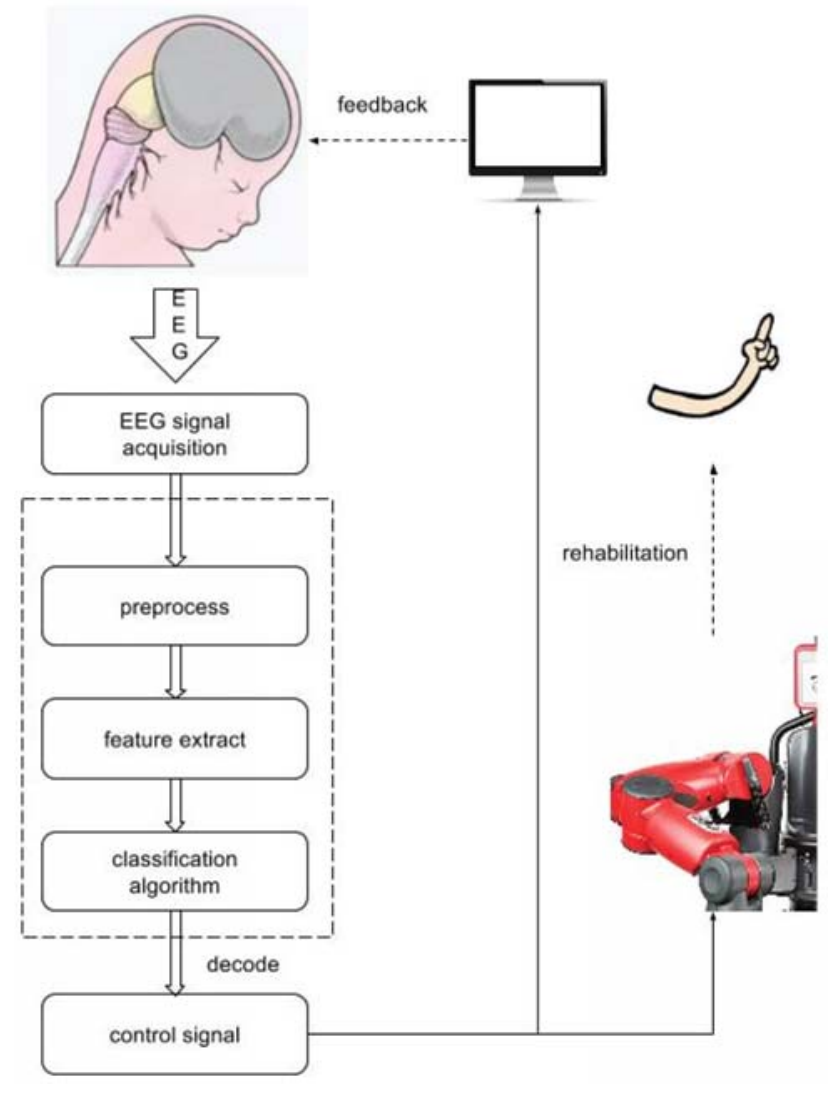

FIGURE II. OUTLOOK OF REHABILITATION SYSTEM

The subjects used an EEG hood to obtain the EEG signal. After we got this signal, we put this signal into the algorithm we discussed above to decode the subject's idea of whether he was thinking about relaxing the arm or contracting The arm. We use the robot arm will be based on the results of this decoding the implementation of the corresponding action, driving the subject into the real arm of the real movement. In this way, the robot arm is equivalent to a very high degree of freedom of the exoskeleton. At the same time, the virtual reality display device will present the current state of the arm on the screen, and feedback the subject of this action when the implementation of the thinking signal.After a long period of traning, we can see that out rehabilitation system will play a significant role in improve the limb movement ability of human body. 
Based on the three criteria of the rehabilitation system disscussed above, we can conclude that out BCI-based rehabilitation system can achieve an effecitve effect on the recovery process of stroke patients.

\section{CONCLUSION}

EEG-based rehabilitation system is the biggest challenges in biomedical engineering. We propose a classification algorithm use features like SCP and FFT, classified by a SVMand a fusion algorithm is used to fuse their classification results. Also we designed a rehabilitation system, it has been proved that such rehabilitation system can help stroke patients.

There are many work to do in the future work. First, we need to design a better signal channels select strategy in automation by compute the entropy of every channel. Second, a high-level feature could be extracted to improve the accuracy of a single classification algorithm. Third, more features and classifiers can be fusion in our fusion framework. In another way, we could collection more train data to train our classifier, extend out EEG classification algorithm to M-class problem, also we can extend the current offline classification algorithm to online classification algorithm to become more realtime.

At last, different BCI systems or BCI and non-BCI systems can be combined to a "hybrid BCI". hybrid BCIs have shown great improvements in several performance criteria such as accuracy and information transfer rate, complexity of the system, and user acceptability.

\section{ACKNOWLEDGMENT}

This work was supported by the National Natural Fund: 91420302 and 91520201.

\section{REFERENCES}

[1] Jonathan R Wolpaw, Niels Birbaumer, Dennis J McFarland, Gert Pfurtscheller, and Theresa M Vaughan. Brain-computer interfaces for communication and control. Clinical neurophysiology, 113(6):767- 791, 2002.

[2] Alexander J Doud, John P Lucas, Marc T Pisansky, and Bin He. Continuous three-dimensional control of a virtual helicopter using a motor imagery based brain-computer interface. PloS one, 6(10):e26322, 2011.

[3] Stephan Waldert, Tobias Pistohl, Christoph Braun, Tonio Ball, Ad Aertsen, and Carsten Mehring. A review on directional information in neural signals for brain-machine interfaces. Journal of Physiology- Paris, 103(3):244-254, 2009.

[4] Herbert Ramoser, Johannes Muller-Gerking, and Gert Pfurtscheller. Optimal spatial filtering of single trial eeg during imagined hand movement. Rehabilitation Engineering, IEEE Transactions on, 8(4):441- 446, 2000.

[5] Guido Dornhege, Benjamin Blankertz, Matthias Krauledat, Florian Losch, Gabriel Curio, and K-R Muller. Combined optimization of spatial and temporal filters for improving brain-computer interfacing. Biomedical Engineering, IEEE Transactions on, 53(11):2274-2281, 2006.

[6] Kai Keng Ang, Zheng Yang Chin, Haihong Zhang, and Cuntai Guan. Filter bank common spatial pattern (fbcsp) in brain-computer interface. In Neural Networks, 2008. IJCNN 2008.(IEEE World Congress on Computational Intelligence). IEEE International Joint Conference on, pages 2390-2397. IEEE, 2008.

[7] Ebrahim A Mousavi, Jerome J Maller, Paul B Fitzgerald, and Brian J Lithgow. Wavelet common spatial pattern in asynchronous offline brain computer interfaces. Biomedical Signal Processing and Control, 6(2):121-128, 2011.

[8] Lotte F, Congedo M, Lécuyer A, et al. A review of classification algorithms for EEG-based brain-computer interfaces[J]. Journal of neural engineering, 2007, 4(2): R1.

[9] Vigário R, Sarela J, Jousmiki V, et al. Independent component approach to the analysis of EEG and MEG recordings[J]. IEEE transactions on biomedical engineering, 2000, 47(5): 589-593.

[10] Lu J, Xie K, McFarland D J. Adaptive spatio-temporal filtering for movement related potentials in EEG-based brain-computer interfaces[J]. IEEE Transactions on Neural Systems and Rehabilitation Engineering, 2014, 22(4): 847-857.

[11] Murugappan M, Murugappan S. Human emotion recognition through short time Electroencephalogram (EEG) signals using Fast Fourier Transform (FFT)[C]//Signal Processing and its Applications (CSPA), 2013 IEEE 9th International Colloquium on. IEEE, 2013: 289-294.

[12] Li Y, Guan C, Li H, et al. A self-training semi-supervised SVM algorithm and its application in an EEG-based brain computer interface speller system[J]. Pattern Recognition Letters, 2008, 29(9): 1285-1294.

[13] Leeb R, Sagha H, Chavarriaga R, et al. A hybrid brain-computer interface based on the fusion of electroencephalographic and electromyographic activities[J]. Journal of neural engineering, 2011, 8(2): 025011.

[14] Angulo-Sherman I N, Costa-García A, Monge-Pereira E, et al. BCI Applied to Neurorehabilitation[M]//Emerging Therapies in Neurorehabilitation II. Springer International Publishing, 2016: 169-196. 